\title{
Fast and Alfvén waves driven by azimuthal footpoint motions
}

\section{Periodic driver}

\author{
A. De Groof, K. Paes, and M. Goossens \\ Centre for Plasma-Astrophysics, K.U. Leuven, Celestijnenlaan 200 B, 3001 Heverlee, Belgium
}

Received 12 November 2001 / Accepted 5 February 2002

\begin{abstract}
The excitation of Alfvén and fast magneto-acoustic waves in coronal loops driven by footpoint motions is studied in linear, ideal MHD. The analysis is restricted to azimuthally polarized footpoint motions so that only Alfvén waves are directly excited which couple to fast magneto-acoustic waves at later times. In the present study a periodic driver is applied at one end of the loop. The effects of a more realistic random driver are studied in the companion paper De Groof \& Goossens (2002) (hereafter referred to as Paper II). The first part of the paper is devoted to the study of resonant absorption and phase-mixing in the absence of coupling (azimuthal wavenumber $\left.k_{y}=0\right)$. Since the density varies across the loop, resonances occur at the magnetic surfaces where the driving frequency equals the local Alfvén frequency. In a second part where Alfvén waves with $k_{y} \neq 0$ coupling to fast waves are taken into account, we find that the behaviour of the MHD waves is strongly dependent on the driving frequency $\omega_{\mathrm{d}}$. Especially driving frequencies equal to a quasi-mode frequency seem to make the difference. The fast waves excited in these cases are global oscillations of the system and form quasi-modes as they are damped through the resonant coupling with Alfvén modes. Since these resonances occur at the same location where the original Alfvén wave peaks, the resonant peak is further amplified. While in most cases coupling has a negative effect on the growth of the directly excited Alfvén waves, driving with a quasi-mode frequency leads to a faster growth of the resonant peaks and a more efficient decrease in length scales than in the uncoupled case.
\end{abstract}

Key words. MHD - Sun: corona - Sun: magnetic fields - waves

\section{Introduction}

Observations of the solar corona by Yohkoh, the Solar and Heliospheric observatory (SOHO) and the Transition Region And Coronal Explorer (TRACE) show that the corona displays a complex and very dynamical system of magnetic structures. Moreover, from X-ray images, we know that the hot coronal plasma of several million degrees is contained in loop-like structures, designed by the strong magnetic field, which lead to the assumption that the heating of the corona has a magnetic nature. The high conductivity and relatively high mass density of the photospheric plasma provide a very effective anchoring of the coronal magnetic field lines in the underlying photosphere ("line-tying"). As a consequence, the photospheric footpoints of a coronal loop are forced to follow the convective motions in the photosphere. Several scenarios have been proposed for the heating of a loop, depending upon how fast the footpoints are moved. For fast motions compared to the Alfvén transit time along the loop, Alfvén

Send offprint requests to: A. De Groof,

e-mail: anik.degroof@wis.kuleuven.ac.be waves and magnetosonic waves are generated and propagate inside the loop. Due to the steep density gradients at the photospheric edges, these waves are reflected back and forth along the length of the loop resulting in a complex interference pattern. This wave energy can be converted to heat when sufficiently steep velocity gradients and small length scales are created by means of resonant absorption (Goossens 1991) or phase-mixing (Heyvaerts \& Priest 1983). The process of dissipation can be further enhanced by turbulence (Gomez 1990).

On the other hand, when the footpoint motions are slow compared to the Alfvén transit time along the loop, the magnetic field lines within the loop are twisted and deformed. The magnetic stresses, which are built up that way, and the small length scales in between fieldlines of different polarity, lead to magnetic reconnection and hence to a conversion of magnetic energy into heat (Parker 1972; Van Ballegooijen 1985). Tsuneta (1996) showed that this type of heating mechanism could explain some coronal phenomena observed by Yohkoh. In addition, magnetic reconnection might be very important to provide the fast footpoint motions needed for wave mechanisms in higher 
coronal loops. Therefore, the question might not be which mechanism to choose, but rather how to combine them. Recent observations suggest that the different heating mechanisms may co-operate: nanoflaring in the chromospheric magnetic network might both directly heat the coronal plasma and generate waves that propagate into extended loops and are dissipated there to provide the extra heat in the bodies of these larger structures (Falconer et al. 1997). Moreover, reconnection events in smaller loops might trigger fast or Alfvén MHD waves propagating in overlaying larger loops, paving the way for dissipation mechanisms like resonant absorption. Observations reveal that most explosive events, typically evolving on time scales ranging from 20 to $200 \mathrm{~s}$, are associated with the emergence of new magnetic flux. Magnetic field annihilations between the emerging and the pre-existing field then probably lead to an acceleration of plasma. Roussev et al. (2001) carried out numerical simulations of explosive events where reconnection has indeed excited and driven fast magneto-acoustic and Alfvén waves.

The dissipation mechanism on which we focus is the process of resonant absorption where a local resonant Alfvén wave is excited by inciding MHD waves. In 1978, Ionson suggested that resonant absorption might effectively heat the corona and since this original suggestion, it has remained a popular mechanism for explaining the coronal heating. Since the frequency of an Alfvén wave is dependent upon the plasma density, there will be a continuum of Alfvén eigenfrequencies in a coronal loop with a varying density. The local Alfvén waves can be excited either by sideways impinging fast MHD waves, due to e.g. an exploding granule in the vicinity of the loop, or by MHD waves generated through footpoint motions at the base of the coronal loop. At the magnetic surface where the frequency of the inciding wave equals the local Alfvén frequency, a resonant Alfvén wave can be excited. The resonant peak resulting from this energy transfer supplies the small lengthscales necessary for dissipation to become effective (Kuperus et al. 1981; Goossens 1991). Numerical results reveal that sideways excitation is most efficient when the loop is driven at the frequency of a fast eigenmode, lying inside the Alfvén continuum, a so-called "quasi-mode" (Poedts et al. 1989; Poedts \& Kerner 1992; Steinolfson \& Davila 1993; Ofman \& Davila 1995; Ofman \& Davila 1996; Wright \& Rickard 1995). Poedts et al. (1994) showed that the resonances display a high rate of Ohmic heating compared to the total energy stored in the loop. This indicates that the time scales of the heating process can be relatively short and resonant absorption turns out to be a good candidate for the heating of the coronal loops.

Besides sideways impinging waves, resonant Alfvén waves can also be excited by fast MHD waves originating from within the loop. As explained by Tirry et al. (1997), these inherent waves are even more likely to be the prime contribution to the heating of the loop by resonant absorption. Such a fast wave can be excited by e.g. a reconnection event inside the loop or in smaller, low-lying loops, or by the photospheric motions of the footpoints of the magnetic field lines. Resonant absorption can be achieved either by directly exciting Alfvén waves with azimuthally polarized footpoint motions or indirectly (through coupling) with radially polarized footpoint motions.

The theoretical investigation of footpoint excitation of MHD waves involves the explicit solution of the wavedynamics not only in the radial direction, but also in the longitudinal direction in order to include the appropriate boundary conditions at the loop's feet. In order to avoid coupling between Alfvén waves and fast waves, authors have often assumed the azimuthal wave number to be zero (Heyvaerts \& Priest 1983; Berghmans \& De Bruyne 1995; Berghmans et al. 1996; Poedts \& Boynton 1996; Ruderman et al. 1997). However more recent studies revealed the importance of coupling both for radially polarized footpoint motions (Tirry et al. 1997; De Groof \& Goossens 2000), as for azimuthally polarized footpoint motions (Berghmans \& Tirry 1997; Tirry \& Berghmans 1997). In the last two papers the authors investigated the interaction between the Alfvén waves directly excited at the footpoints and the Alfvén waves indirectly excited through coupling with fast MHD waves. They found that the presence of quasi-modes has an important influence on the dissipation; for a harmonic driver, the resonances built up by the resonant Alfvén waves are either amplified or opposed by the indirect excited waves. In the limiting case where the two resonances exactly oppose each other, a "zero-resonance-line" is found (Tirry \& Berghmans 1997). In Goossens \& De Groof (2001), the most important results concerning resonant and phase-mixed MHD waves excited by footpoint motions are brought together.

Obviously, all of the models used in theoretical investigations are hampered by assumptions. Therefore it is important to focus on one aspect of the problem and adjust the model so that the quality of the results is maximally guaranteed. In principle, theoretical studies of wave heating due to footpoint motions should solve the time dependent nonlinear dissipative MHD equations in the presence of boundary motions for a realistic equilibrium model. The equilibrium model of the loop should include transversal and longitudinal variations (as discussed above) and the forcing should be a realistic representation of the boundary motions which should not be restricted to a particular type of motions. A theoretical study based on a realistic two-dimensional equilibrium model and allowing for an unrestricted choice of the boundary motions has not yet been carried out. Most of the earlier investigations, so as the present one, are carried out in cylindrical or slab equilibrium models with only stratification in the radial direction, without taking into account the stratification caused by the chromosphere and transition region. However, Ofman et al. (1998) and Beliën et al. (1999) revealed that the implementation of the chromosphere and transition layer at the base of the loop has an important influence on the efficiency of resonant absorption. Beliën et al. included the chromospheric and coronal density scale heights and found a lower efficiency of resonant absorption 
than in equivalent line-tied loop systems. The loop model they used is two-dimensional and a good approximation of reality. For the boundary motions, however, the authors made a very particular and restrictive choice as they applied torsional Alfvén waves at the magnetic footpoints. Torsional Alfvén waves have $m=0$ or $k_{y}=0$ and do not couple to magnetosonic MHD waves in the linear regime. An important result Beliën et al. found in their numerical outcome is a considerable transfer of energy from the Alfvén waves to compressional, slow magneto-acoustic oscillations, non-linearly generated in the chromosphere and transition region. Therefore they concluded that the coronal heating by resonant absorption of Alfvén waves is much less efficient than the indirect heating through the non-linear generation of the compressional magnetoacoustic oscillations. Now this conclusion is reached for boundary motions which in the linear regime are uncoupled to compressional waves and only weakly coupled in the nonlinear regime. The obvious conclusion is that the driving should not be restricted to torsional Alfvén waves but should be a combination of Alfvén and compressional MHD waves coupled to each other through a non-zero azimuthal wave number so that all significant wave modes are taken into account. Apart from this, the implementation of a monochromatic driver is rather unfortunate in the simulations of Beliën et al. since the length of the coronal loops varies continuously due to shifts of the chromosphere and consequently detuning of the loops is unavoidable if only one frequency is present in the driving spectrum.

Ofman et al. on the other hand used a simplified model to implement the effect of the chromosphere but they took into account both coupling and a broadband driver. They found that the time dependence of the density due to plasma flows in and out the corona leads to multiple resonance layers drifting throughout the loop to heat the entire volume. Since they only performed simulations with a fixed $k_{z}$, the amount of resonant layers was still limited to the ones corresponding to the dominant quasi-mode. The papers by Beliën et al. and Ofman et al. are both important but have their limitations. Either only torsional Alfvén waves could be studied because of the complicated equilibrium model, as in the former paper, or the driver is more realistic but not all natural modes, important for the generation of small length scales, are taken into account.

In the present paper, we continue the time-dependent study of Alfvén and fast waves driven by azimuthally polarized footpoint motions, started by Tirry \& Berghmans (1997). We focus on the interaction between Alfvén waves and compressible MHD waves in its full extent right from the linear regime. In this sense, the present paper is complementary to Beliën et al. and Ofman et al.. Moreover we aim to stress the importance of taking into account the whole set of quasi-modes $\left(k_{z} \neq\right.$ constant $)$ since quasimodes play an important role in coronal loops driven by azimuthal footpoint motions, as investigated before for radial motions (De Groof \& Goossens 2000). Adding stratification in the axial direction might alter some properties of the investigated MHD waves and their intrinsic coupling but the overall properties found in one-dimensional equilibrium models will not be lost. Besides, in case the coronal loops are directly driven by magnetic reconnection events in the chromosphere and lower corona, the stratification at the base of the loop is less important.

The implementation of a periodic driver at one of the footpoints obviously leads to an important simplification too. Nevertheless, it will give us a clear picture of the physics behind resonant absorption and phase-mixing and it allows us to investigate the importance of the quasimodes and the influence of the strength of the coupling. In Paper II a more realistic, random driver is studied.

The paper is organized as follows. In the next section the physical model and the relevant equations and boundary conditions are discussed. In Sect. 3 we repeat the important steps in the derivation of the analytical solution, which describes the temporal behaviour of the excited MHD waves, as given by Tirry \& Berghmans (1997). The solution is written as a superposition of eigenmodes. In Sect. 4, the meaning and importance of the "quasimodes" is clarified. The role they play in coronal heating is shown in Sect. 5. In the first part 5.1, the mechanisms of resonant absorption and phase-mixing are studied in the absence of coupling $\left(k_{y}=0\right)$. In Sect. 5.2 coupling is taken into account and we investigate how the behaviour of the MHD waves depends on the driving frequency $\omega_{\mathrm{d}}$ and the value of $k_{y}$. Finally in Sect. 6 we give a summary and discussion.

\section{Physical model}

We model the coronal loop as a static, straight, gravitationless plasma slab with thickness $b$, obeying the standard set of ideal MHD equations (Fig. 1). In our Cartesian coordinate system, the $x$-coordinate corresponds to the direction of the inhomogeneity in the equilibrium, the $y$ coordinate is the (ignorable) azimuthal coordinate and the $z$-coordinate represents the direction along the loop.

At $z=0$ we impose azimuthally polarized footpoint motions whereas at $z=L$ we assume the loop to be linetied. The plasma is permeated by a uniform magnetic field $\left(\boldsymbol{B}_{0}=B_{0} \boldsymbol{e}_{z}\right)$ and has a uniform pressure $p_{0}$ which we neglect in comparison with the magnetic pressure ("zerobeta-approximation"). The inhomogeneity of the plasma is introduced by a continuously varying density

$\rho_{0}(x)=\rho_{\mathrm{A}}+\rho_{\mathrm{B}} \cos \left(\frac{\pi}{b} x\right) \quad$ with $\quad \rho_{\mathrm{B}}<\rho_{\mathrm{A}}$,

which models the higher density inside the loop.

The plasma is being shaken by small-amplitude perturbations at the footpoints of the magnetic field lines on the $z=0$ plane. As long as non-linear and non-ideal effects are negligible we can follow the temporal evolution of the excited MHD waves inside the loop by solving the linear ideal MHD equations. Since the equilibrium quantities are constant in the $y$-coordinate which runs over an infinite interval, we can Fourier analyse with respect to $y$. For the Fourier component corresponding to wave number 


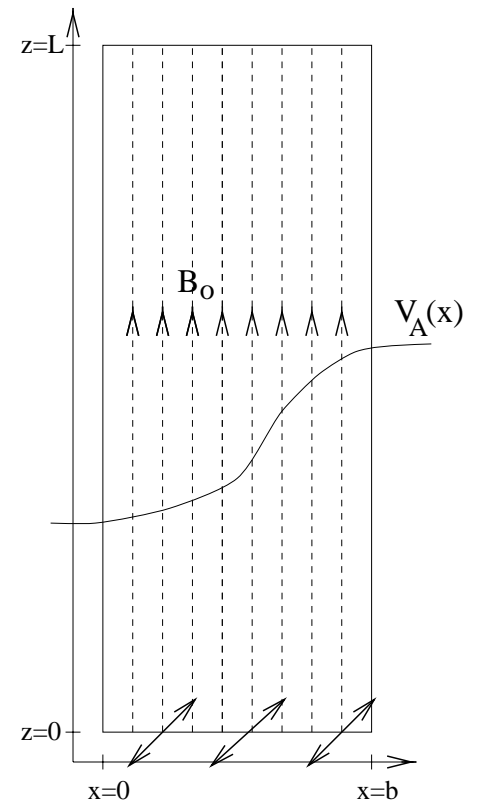

Fig. 1. Cartoon model of the coronal loop driven by azimuthally polarized footpoint motions.

$k_{y}$, the time evolution and the spatial variation in $x$ and $z$ are described by

$$
\begin{aligned}
& \left\{\frac{1}{v_{\mathrm{A}}^{2}} \frac{\partial^{2}}{\partial t^{2}}-\frac{\partial^{2}}{\partial z^{2}}-\frac{\partial^{2}}{\partial x^{2}}\right\} \xi_{x}=i k_{y} \frac{\partial \xi_{y}}{\partial x}, \\
& \left\{\frac{1}{v_{\mathrm{A}}^{2}} \frac{\partial^{2}}{\partial t^{2}}-\frac{\partial^{2}}{\partial z^{2}}+k_{y}^{2}\right\} \xi_{y}=i k_{y} \frac{\partial \xi_{x}}{\partial x},
\end{aligned}
$$

where $\boldsymbol{\xi}$ is the Lagrangian displacement and the Alfvén speed $v_{\mathrm{A}}$ is given by

$$
v_{\mathrm{A}}(x)=\sqrt{\frac{B_{0}^{2}}{\rho_{0}(x)}} .
$$

From Eqs. (2) and (3) it is clear that $k_{y}$ can be seen as a coupling factor that controls the coupling between the fast waves, characterized by $\xi_{x}$ and the Alfvén waves, characterized by $\xi_{y}$. For $k_{y}=0$ Alfvén and fast waves are linearly decoupled and there is only a weak coupling in the nonlinear regime. Slow waves are absent $\left(\xi_{z}=0\right)$ because the plasma pressure is neglected.

When $k_{y} \neq 0$ is taken to be non-zero, the azimuthally polarized footpoint motions indirectly drive fast MHD waves which couple to Alfvén waves at the resonant surfaces where the ideal Alfvén wave resonance condition is satisfied (Mann \& Wright 1995). Length, speed, magnetic field strength and density are non-dimensionalized with respect to $b, v_{\mathrm{A}}(0), B_{0}$ and $\rho(0)$ respectively.

\section{Mathematical method}

In this section we derive a formal analytical solution of the coupled Eqs. (2) and (3) which describes the temporal evolution of the excited linear MHD waves. Since the derivation is analogous to the method given by
Tirry et al. (1997), we confine the description of the mathematical method to its most important steps. The analysis is originally based on the matrix eigenvalue method derived by Mann et al. (1995) (numerical side of the derivation) and on the paper by Berghmans et al. (1996) (analytical approach of footpoint motions).

The azimuthally polarized footpoint motions are represented by inhomogeneous boundary conditions for Eqs. (2) and (3) at the $z=0$ and the $z=L$ boundary planes:

$\xi_{x}(x, z=0, t)=0$,

$\xi_{x}(x, z=L, t)=0$,

$\xi_{y}(x, z=0, t)=R(x) T(t)$,

$\xi_{y}(x, z=L, t)=0$.

We have assumed for mathematical simplicity that the dependencies on $x$ and $t$ of the footpoint motions are separable. We assume in addition that at $t=0, \xi_{x}, \xi_{y}$ and both their time derivatives are zero and as a consequence:

$T(t=0)=\frac{\partial T(t=0)}{\partial t}=0$.

With the aid of the function

$\varphi(x, z, t)=i \xi_{y}(x, z, t)-\left(1-\frac{z}{L}\right) R(x) T(t)$,

we include the footpoint motions as driving terms in the equations, while the boundary conditions become homogeneous:

$\left\{\frac{1}{v_{\mathrm{A}}^{2}} \frac{\partial^{2}}{\partial t^{2}}-\frac{\partial^{2}}{\partial z^{2}}-\frac{\partial^{2}}{\partial x^{2}}\right\} \xi_{x}=k_{y} \frac{\partial \varphi}{\partial x}+\left(1-\frac{z}{L}\right) k_{y} \frac{\partial R}{\partial x} T$,

$\left\{\frac{1}{v_{\mathrm{A}}^{2}} \frac{\partial^{2}}{\partial t^{2}}-\frac{\partial^{2}}{\partial z^{2}}+k_{y}^{2}\right\} \varphi=-k_{y} \frac{\partial \xi_{x}}{\partial x}-\left(1-\frac{z}{L}\right) k_{y}^{2} R T$

$$
-\left(1-\frac{z}{L}\right) \frac{1}{v_{A}^{2}} \frac{\partial^{2}}{\partial t^{2}} R T
$$

and

$\xi_{x}(z=0)=0=\xi_{x}(z=L), \quad \varphi(z=0)=0=\varphi(z=L)$.

By means of sine-expansions for $\xi_{x}$ and $\varphi$, we are able to rewrite the coupled partial differential Eqs. (7) and (8) as an infinite set of coupled partial differential equations for $X^{(n)}$ and $Y^{(n)}$ :

$\left\{\frac{1}{v_{\mathrm{A}}^{2}} \frac{\partial^{2}}{\partial t^{2}}+\left(\frac{n \pi}{L}\right)^{2}-\frac{\partial^{2}}{\partial x^{2}}\right\} X^{(n)}$

$$
=k_{y} \frac{\partial Y^{(n)}}{\partial x}+\frac{L}{n \pi} k_{y} \frac{\partial R}{\partial x} T
$$

$$
\begin{aligned}
\left\{\frac{1}{v_{\mathrm{A}}^{2}} \frac{\partial^{2}}{\partial t^{2}}+\right. & \left.\left(\frac{n \pi}{L}\right)^{2}+k_{y}^{2}\right\} Y^{(n)} \\
& =-\frac{L}{n \pi}\left(k_{y}^{2}+\frac{1}{v_{\mathrm{A}}^{2}} \frac{\partial^{2}}{\partial t^{2}}\right) R T-k_{y} \frac{\partial X^{(n)}}{\partial x}
\end{aligned}
$$

With the aid of the Laplace transformation, we find two coupled ordinary differential equations for each $n$ :

$\left\{A[n]-I \omega^{2}\right\}\left[\begin{array}{l}\hat{X}^{(n)} \\ \hat{Y}^{(n)}\end{array}\right]=B[n]$ 
where

$$
\begin{gathered}
A[n]=v_{\mathrm{A}}^{2}\left[\begin{array}{cc}
\left(\frac{n \pi}{L}\right)^{2}-\frac{\partial^{2}}{\partial x^{2}} & -k_{y} \frac{\partial}{\partial x} \\
k_{y} \frac{\partial}{\partial x} & \left(\frac{n \pi}{L}\right)^{2}+k_{y}^{2}
\end{array}\right], \\
B[n]=\frac{L}{n \pi} v_{\mathrm{A}}^{2} \hat{T}\left[\begin{array}{c}
k_{y} \frac{\partial R}{\partial x} \\
-k_{y}^{2} R(x)+\frac{\omega^{2}}{v_{\mathrm{A}}^{2}} R(x)
\end{array}\right],
\end{gathered}
$$

and $I$ is the unity matrix.

Equation (11) can be solved by inverting the operator $A[n]-I \omega^{2}$, in the same way as done by Tirry et al. (1997) and inspired by Cally (1991). After inverting the transformations, we now can reconstruct the displacement components $\xi_{x}$ and $\xi_{y}$ as functions of $x, z$ and $t$

$$
\begin{aligned}
& \xi_{x}(x, z, t)=\frac{2}{L} \sum_{n=1}^{\infty} \\
& \sum_{m=1}^{N}\left\{\frac{n \pi}{L} \sum_{k=1}^{2 N+1} \mathcal{R}_{k}^{n} \mathcal{T}_{k}^{n}(t) \alpha_{m k}^{n}\right\} \sin (m \pi x) \sin \left(\frac{n \pi}{L} z\right) \\
& i \xi_{y}(x, z, t)=\frac{2}{L} \sum_{n=1}^{\infty} \frac{n \pi}{L}\left\{\sum_{k=1}^{2 N+1} \mathcal{R}_{k}^{n} \mathcal{T}_{k}^{n}(t) \frac{\beta_{0 k}^{n}}{2}\right. \\
& \left.+\sum_{m=1}^{N}\left(\sum_{k=1}^{2 N+1} \mathcal{R}_{k}^{n} \mathcal{T}_{k}^{n}(t) \beta_{m k}^{n}\right) \cos (m \pi x)\right\} \sin \left(\frac{n \pi}{L} z\right) \\
& +R(x) T(t)\left(1-\frac{z}{L}-\frac{2}{L} \sum_{n=1}^{\infty} \frac{L}{n \pi} \sin \left(\frac{n \pi}{L} z\right)\right),
\end{aligned}
$$

where

$\mathcal{R}_{k}^{n} \equiv \int_{0}^{1} R(x) \mid \psi_{k}^{n}(x)>\mathrm{d} x$,

$\mathcal{T}_{k}^{n}(t) \equiv \frac{1}{\omega_{k}} \int_{0}^{t} \sin \left(\omega_{k}(t-\tau)\right) T(\tau) \mathrm{d} \tau$.

The last term of Eq. (13) equals zero for all positive values of $z$. However when $z=0$ this term equals $R(x) T(t)$ which is indeed the imposed azimuthally polarized footpoint motion.

Thus we have derived an analytical expression which describes the generation of linear MHD waves (coupled fast-Alfvén waves) by azimuthally polarized footpoint motions. The solution is written as a superposition of eigenmodes $\mid \psi_{k}^{n}>$ whose excitation is determined by the time dependence $T(t)$ of the footpoint motion through the convolution $\mathcal{T}_{k}^{n}$ and by the spatial dependence $R(x)$ of the footpoint motion through the scalar product $\mathcal{R}_{k}^{n}$. This expression can easily be evaluated numerically at any time with the structure of the waves fully resolved as long as a sufficiently large numbers of sines in both $x-$ and $z$-directions are taken into account.

\section{Significance of the so-called quasi-modes}

When a coronal loop is driven, the nature of its response is determined by its spectrum of eigenoscillations. In our model, in which the slow waves are removed by the assumption of $\beta=0$, the spectrum consists of two parts. The first part is the fast magnetosonic spectrum. It is a series of discrete, fast eigenmodes, oscillating with the same (eigen)frequency throughout the whole loop. The second part of the spectrum is the Alfvén spectrum, governed by

$\left\{\rho \omega_{\mathrm{A}}^{2}+\frac{\partial^{2}}{\partial z^{2}}\right\} \xi_{y}=0$,

where $\omega_{\mathrm{A}}$ is the local Alfvén frequency $\omega_{\mathrm{A}}^{2}(x)=k_{z}^{2} v_{\mathrm{A}}^{2}(x)$. Because of the spatial dependence of this local Alfvén frequency, a continuum of resonant Alfvén waves is defined. In case there are no boundary conditions in the $z$-direction, which is assumed in many related papers, there is one Alfvén continuum related to a specified $k_{z}$. For a line-tied loop, like the model we use here, the situation is more involved. As seen from expressions (12) and (13) which describe the solutions as a superposition of eigenmodes, the possible values of $k_{z}$ are multiples of $\pi / L$ $\left(k_{z}=\frac{n \pi}{L}\right)$. Consequently, with each $n$ a set of equations is associated and an infinite number of Alfvén continua appears. Figure 2 shows the eigenfrequencies of the first three fast eigenmodes together with the upper and lower bound of the Alfvén continuous spectrum as function of $k_{z}$.

For small values of $k_{y}$ (see De Groof \& Goossens 2000), fast modes corresponding to an eigenfrequency above the Alfvén continuum (leaky modes) are propagating waves in the exterior coronal environment and consequently they radiate their energy away from the loop. Their description could be problematic in the "closed-box model" we adopt in our calculations. However, in the case of azimuthally polarized footpoint motions, global fast modes cannot be excited directly but only through coupling to Alfvén waves. Therefore the excited fast waves always have frequencies inside the Alfvén continuum and consequently only "body modes", keeping the energy inside the loop, are present in the system. For $k_{y} \neq 0$ these body modes couple to localized Alfvén waves and form essentially quasi-modes (Tirry \& Goossens 1996). Due to the resonant coupling, small length scales are generated around the resonant point which are necessary for dissipation and hence heating of the coronal plasma.

\section{Importance of quasi-modes for the resonance growth}

As shown in several previous simulations, the presence of quasi-modes in coronal loops seems to have a major influence on the efficiency of wave dissipation as coronal heating mechanism. Tirry et al. (1997) and De Groof \& Goossens (2000) already studied the importance of quasimodes for resonant absorption after respectively a periodic and a random driver, polarized in the radial direction. Since only fast waves are driven directly in that case, quasi-modes are essential for resonant Alfvén waves to be excited and small length scales to be formed. For a random driver we found that resonant peaks are formed at multiple magnetic surfaces spread throughout the loop and 


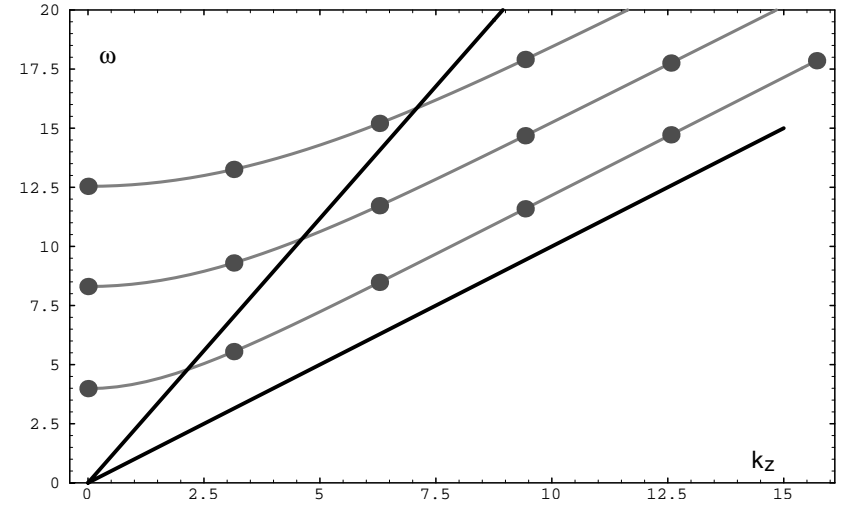

Fig. 2. The eigenfrequencies of the first three fast eigenmodes (gray lines) together with the upper and lower bound of the Alfvén continuum (black lines) as function of $k_{z}(L=1)$.

consequently resonant absorption is a viable candidate to heat the presented loop model in a global way.

On the other hand, azimuthally polarized footpoint motions directly drive resonant Alfvén waves and as a consequence quasi-modes are not strictly necessary for resonant absorption to be efficient. One can even expect the coupling to fast waves to have a negative influence on the growth of Alfvén resonances since indirectly excited Alfvén waves (through coupling with a quasi-mode) could be out of phase with the original resonant Alfvén wave. Berghmans \& Tirry (1997) investigated the stationary state in the coupled case and found that the resonances built up by the resonant Alfvén waves are either amplified or opposed by the indirect excited waves, depending on the driving frequency and the value of $k_{y}$. In addition, they found a so-called "anti-resonance line" at which no heating occurs at all although the loop is driven with a frequency within the Alfvén continuum. In the accompanied paper Tirry \& Berghmans (1997), the same phenomena are investigated using a time-dependent, ideal approach.

As a first step to a full investigation for a more realistic driver (Paper II), we study in the following section the behaviour of the directly and indirectly excited Alfvén modes and the fast magneto-acoustic modes in time. In contrast to the approach of Tirry \& Berghmans (1997), we study the Alfvén mode energy as a whole in order to investigate the influence of the quasi-modes on the growth of the Alfvén resonances. We first recover the results for decoupled Alfvén waves as a test but also as a starting point for our investigation on how this physics is altered when coupling is taken into account.

\subsection{Torsional Alfvén waves}

When purely azimuthal footpoint motions are considered in the uncoupled case $\left(k_{y}=0\right)$, only torsional Alfvén waves are generated, propagating along the magnetic field lines with the local Alfvén speed. In Fig. 3 the displacement $\xi_{y}$ (corresponding to the Alfvén waves) is plotted right after the start of the footpoint motions. We impose

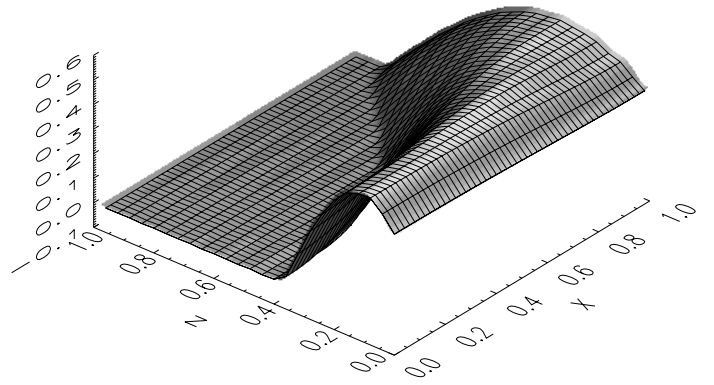

Fig. 3. The Alfvén amplitude $\xi_{y}$ at $t=0.4$ after a periodic driver with $\omega_{\mathrm{d}}=6\left(k_{y}=0\right)$.

footpoint motions with characteristics

$T(t)=\sin (\omega t) \quad R(x)=1$

which correspond to a periodic driver with no $x$ dependence: waves are excited with a constant strength over the whole width of the loop. In Fig. 3 the first wave front is visible, moving in the $z$-direction. The $x$ dependence of the Alfvén speed, caused by the varying density, is in perfect agreement with the density profile introduced in Eq. (1) with $\rho_{\mathrm{A}}=0.6$ and $\rho_{\mathrm{B}}=0.4$.

At later times this wave front is reflected at the rigid wall at $z=1$ and interferes with the following wave fronts, all propagating back and forth in the loop. At the magnetic surface where the local Alfvén frequency matches the driving frequency, the amplitude grows unboundedly due to constructive interference. Close to this resonance point, the Alfvén speed is only slightly different and as a consequence the Alfvén waves on neighbouring surfaces shift out of phase. This phase-mixing process is an extra mechanism to create the small length scales necessary for dissipation to become effective in the solar corona.

The resonance and phase-mixing processes in case of a periodic driver are both visible in Fig. 4, showing the effects of the $x$-dependence of the amplitude of $\xi_{y}$ for a driving frequency $\omega_{\mathrm{d}}=6$. One resonant peak is formed around $x=0.8$, and close to this surface smaller peaks are formed by the phase-mixing process. The amplitude of $\xi_{y}$ clearly follows a smoothed $1 / x$ profile at $t=40$ which in fact is alternated with a smoothed $\delta$ function in time. This behaviour was predicted earlier by Goossens et al. (1995) in dissipative MHD. Since the ideal case of pure $1 / x$ and $\delta$ profiles is only reached at $t=\infty$, Fig. 4 shows smoothed profiles like in dissipative MHD.

The position of the resonance can be predicted (and checked) by rewriting the dispersion relation for Alfvén waves

$\omega_{\mathrm{A}}=\frac{k_{z} B_{0}}{\sqrt{\mu_{0} \rho}}=\frac{n \pi}{\sqrt{\rho}}, \quad n=1,2, \ldots$

where $B_{0}, \mu_{0}$ and $L$ are again set to 1 , to get

$x_{\mathrm{A}}=\frac{1}{\pi} \cos ^{-1}\left(\frac{1}{\rho_{B}}\left(\frac{n^{2} \pi^{2}}{\omega^{2}}-\rho_{\mathrm{A}}\right)\right)$.

Implementing $\omega=6$ for $n=1$ gives indeed $x=0.80$ which 


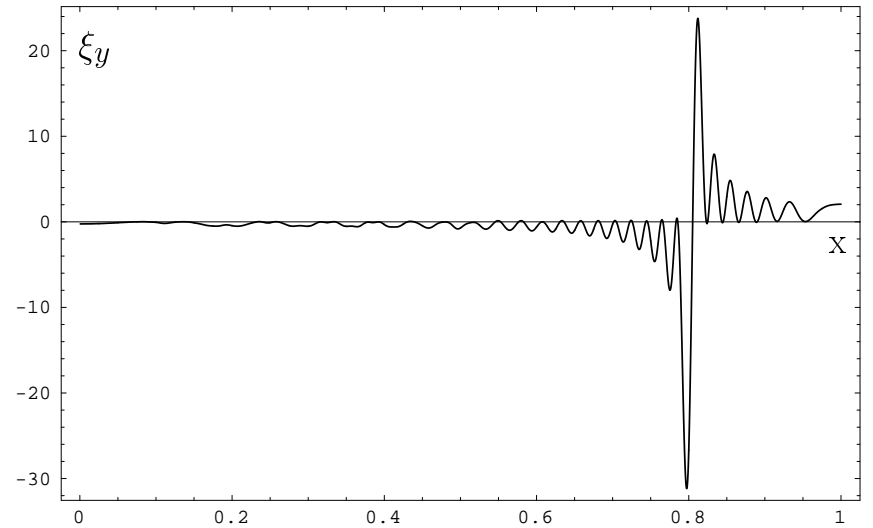

Fig. 4. The amplitude of $\xi_{y}$ at $t=40$, after a periodic driver with driving frequency $\omega_{\mathrm{d}}=6$.

explains the position of the resonant peak in the previous figure. Since for $L=1$ the eigenmodes corresponding to higher values of $n$ lie outside the Alfvén continuum (see Fig. 2), we do not get any other resonant peaks.

For some values of $\omega$ several resonance peaks can be found. For instance the driving frequency $\omega_{\mathrm{d}}=11.5$ gives rise to two resonant surfaces, corresponding to modes with respectively one node $(n=2)$ and two nodes in $z(n=$ 3 ). Indeed, implementing $\omega=11.5$ for $n=2$ gives $x=$ 0.77 while for $n=3$, the resonant surface is at $x=0.44$. In Fig. 5 we plot the resonant peaks and phase-mixing phenomena for $\omega_{\mathrm{d}}=11.5$ in three figures: a 3D-plot, a contour plot and the corresponding cut at $z=0.25$.

For $t \rightarrow \infty$, the amplitude of $\xi_{y}$ on the resonant surfaces is growing unboundedly because of the absence of dissipation in ideal MHD. The neighbouring surfaces, close to the resonance, should show beat phenomena as predicted by theory. That is indeed what we find: in Fig. 6, we plot the beats in the time evolution of $\xi_{y}$ at the nonresonant surface $x=0.75$, where the local Alfvén frequency $\omega_{\mathrm{A}}(x=0.75)=5.58\left(\omega_{\mathrm{d}}=6\right)$. The beat frequency $\left(\omega_{\mathrm{d}}-\omega_{\mathrm{A}}\right) / 2$, analytically found by e.g. Berghmans \& De Bruyne (1995), shows up clearly.

\subsection{The influence of coupling to fast waves}

For $k_{y} \neq 0$, Alfvén waves do no longer exist independently from fast waves. As a consequence the azimuthal driver indirectly drives fast waves through coupling with the Alfvén waves. As shown by Tirry \& Berghmans (1997), the strength of the resonance in the coupled case depends strongly on the driving frequency. In particular, the phase difference beween the directly (footpoints) and indirectly (sideways) excited Alfvén waves and the efficiency of these two ways of driving determine whether the coupling to the fast waves has a positive or negative influence on the amplitude growth at the resonant surface. For some combinations of $k_{y}$ and $\omega_{\mathrm{d}}$ even situations can be found where the two kinds of Alfvén waves have exactly the same amplitude but an opposite phase, so that there is actually no resulting resonance or heating.
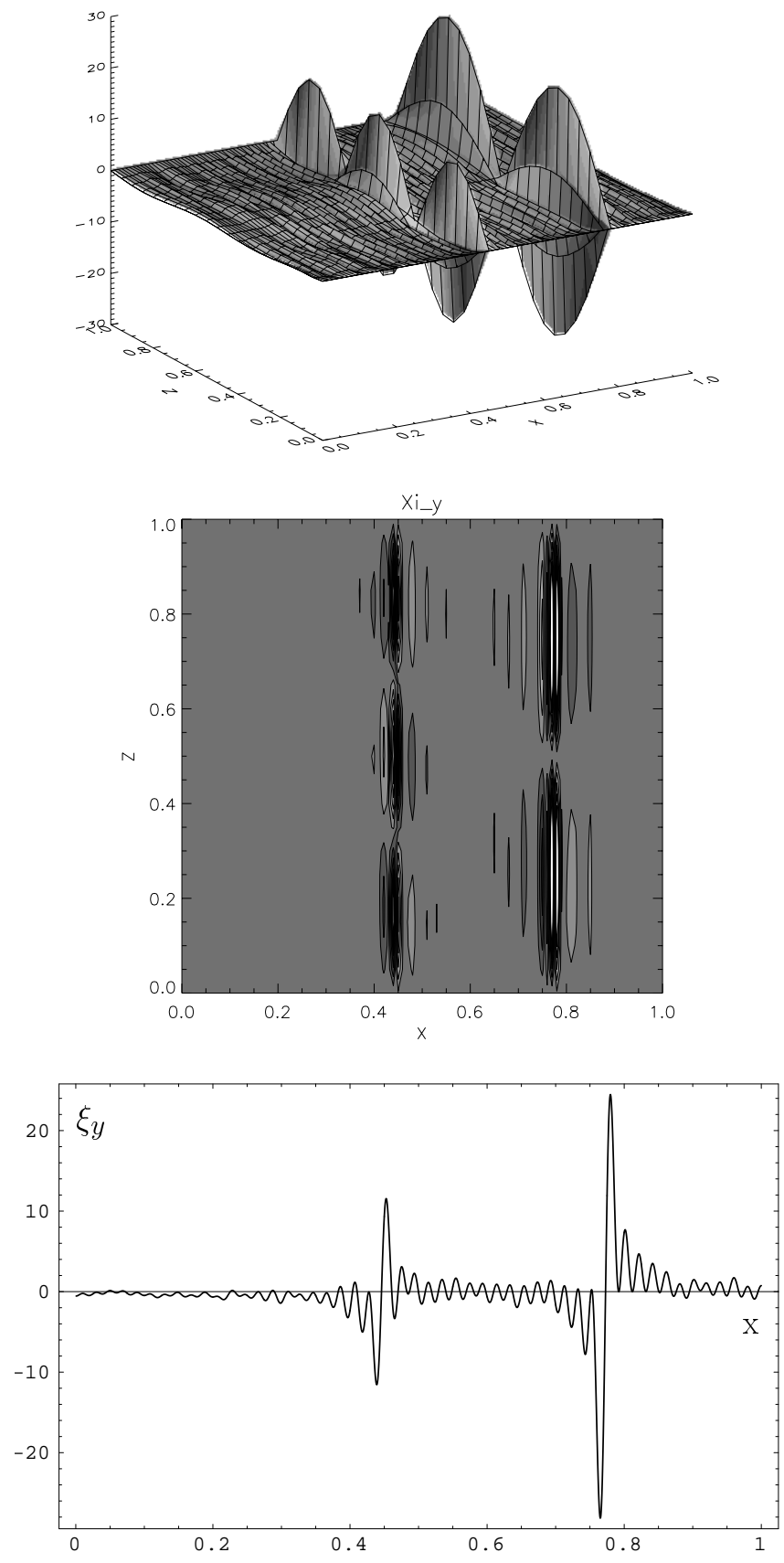

Fig. 5. The amplitude of $\xi_{y}$ at $t=40$, after a periodic driver with driving frequency $\omega_{\mathrm{d}}=11.5$. Top figure: 3D-plot of resonant peaks; middle: corresponding contour plot; bottom figure: cut at $z=0.25$.

In what follows, we do not separate the direct and indirect driven motions but we only investigate the resulting effect of both types of Alfvén waves.

First, when $\omega_{\mathrm{d}}$ is far from any quasi-mode frequency (e.g. $\omega_{\mathrm{d}}=3.5$ ), a resonance is built up at the location where $\omega_{\mathrm{d}}$ equals the local Alfvén frequency (Fig. 7) but it is far less pronounced than in the case of a driver with a quasi-mode frequency (Fig. 8). (The vertical lines represent the resonant positions predicted by theory.)

This difference was to be expected since the small length scales in Fig. 8 show the summed effect of two 


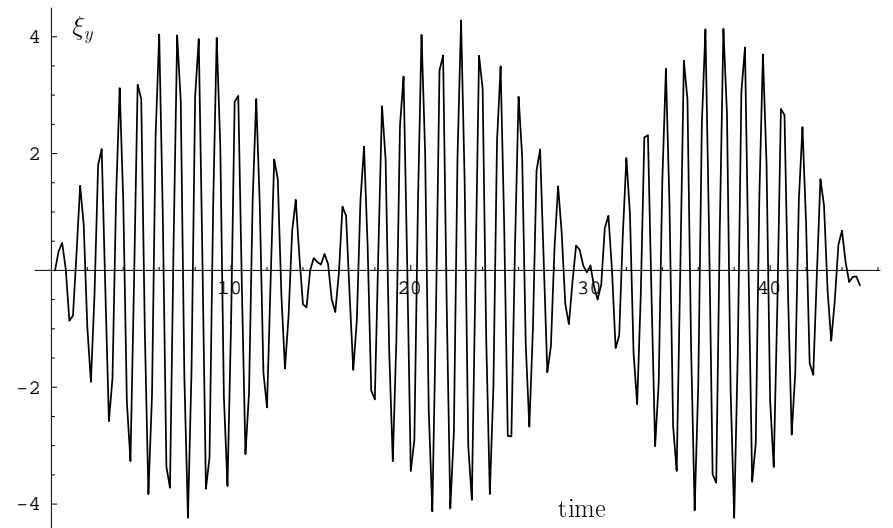

Fig. 6. The amplitude of $\xi_{y}$ at a surface close to the resonant surface $(x=0.75)$ for $k_{y}=0$ with $\omega_{\mathrm{d}}=6$.
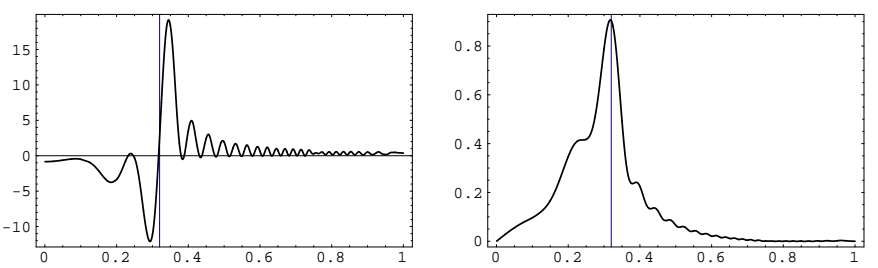

Fig. 7. The amplitude of $\xi_{y}$ and $\xi_{x}$ at $t=40$ as a function of $x$ for $k_{y}=1$ with a driving frequency $\omega_{\mathrm{d}}=3.5$ far from any quasi-mode.

resonances. First, at the considered $x$-location the driving frequency exactly matches the local Alfvén frequency which results in the excitation of a resonant Alfvén wave. Secondly, driving with a quasi-mode frequency, gives rise to the excitation of a fast wave which is characterized by an eigenfrequency of the system. This standing wave in its turn resonantly excites an Alfvén wave at the original resonant surface so that the first resonance is amplified.

The presence of quasi-modes plays an important role in the interplay between fast and Alfvén waves and consequently in the growth of the Alfvén resonance. To investigate these effects, we plot the growth of Alfvén and fast waves as a function of time. For the uncoupled situation $\left(k_{y}=0\right)$, no fast waves are excited $\left(\xi_{x}=0\right)$ and consequently $\xi_{y}$ grows linearly in time. When $k_{y} \neq 0$ however, fast waves enter the system and we get an interplay between the $\xi_{x}$ and $\xi_{y}$ components. Depending on the driving frequency and the value of $k_{y}$ this coupling can have a positive or negative effect on the growth of the $\xi_{y}$-component.

First, for driving frequencies far from any quasi-mode, no global fast waves can be build up, even if the azimuthal wavenumber is non-zero. The driving frequency does not correspond to any eigenmode of the system and consequently the excited fast waves will slowly disappear due to destructive interference. As a result, the amplitude growth at the resonant surface is less effective in case of a stronger coupling, i.e. larger values of $k_{y}$. In Fig. 9, the time evolution of the resonance growth is plotted for $k_{y}=0$ (gray) and $k_{y}=2$ (black) for $\omega_{\mathrm{d}}=3.4$. The graph corresponding to $k_{y}=1$ is only slightly different (slightly less effective)
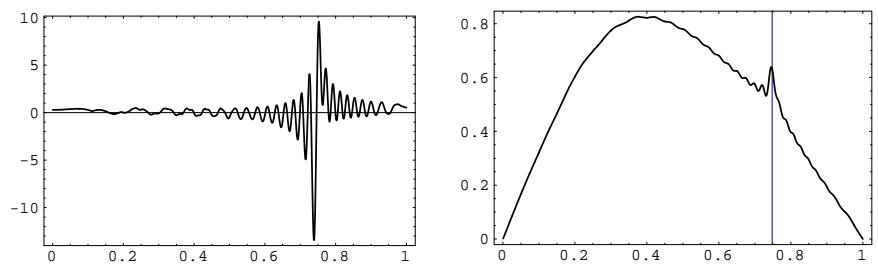

Fig. 8. The amplitude of $\xi_{y}$ and $\xi_{x}$ at $t=40$ as a function of $x$ for $k_{y}=1$ after driving with the quasi-mode frequency $\omega_{\mathrm{d}}=5.52$.

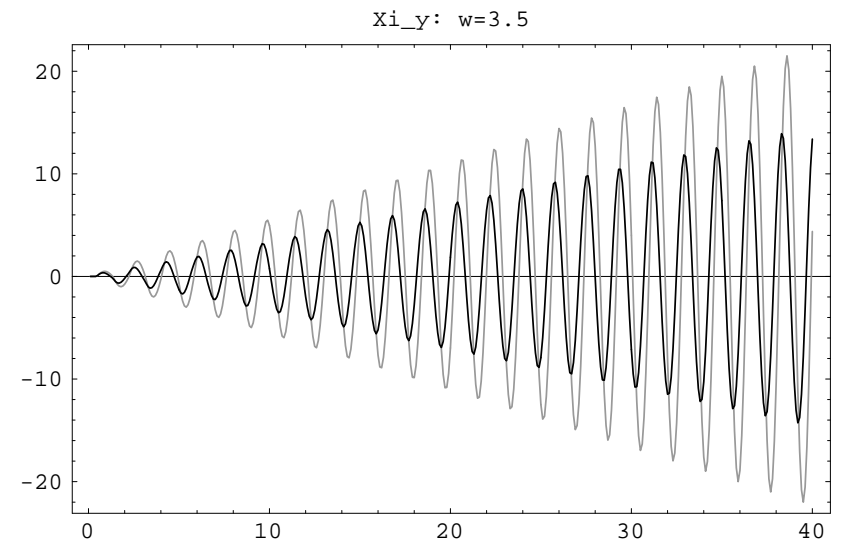

Fig. 9. Time evolution of the amplitude of $\xi_{y}$ for $k_{y}=0$ (gray) and $k_{y}=2$ (black) with driving frequency $\omega_{\mathrm{d}}=3.5$.

than that for $k_{y}=0$ (the uncoupled case) and therefore it is not included in the figure.

The situation is totally different when the system is driven with a quasi-mode frequency. In Fig. 10, we show the time evolution of $\xi_{y}$ and $\xi_{x}$ after $\omega_{\mathrm{d}}=8.51$ for three different values of $k_{y}$. For $k_{y}=0$ (black), we only plot the envelop of the $y$-displacement to keep the figure transparent. Again the linear growth is clear, as well as the absence of the $\xi_{x}$-component. When $k_{y}=1$ (dark-gray), fast waves are excited and we get an interplay between the $x-$ and $y$-components of the displacement. The quasimode looses its energy towards the resonance in correspondence with its damping rate. This means that the $\xi_{x}$ component evolves to a stationary amplitude, as shown in Fig. 10b. As long as no stationary state is reached, the growth of the Alfvén resonance is limited by the loss of energy to the fast waves. Therefore, the resonant peak does not grow as efficiently as in the uncoupled case. However, for a stronger coupling with $k_{y}$ equal to 2 (light-gray), the stationary state sets in much earlier (around $t=30$ ). From that time on the Alfvén resonance grows on a linear scale, with a slope which is steeper than for $k_{y}=0$ so that the resonance growth becomes more efficient than in the uncoupled case.

We get a similar result for the $(n=1)$-quasi-mode, $\omega \approx 5.52$ as shown in Fig. 11a. (Actually, the frequency of quasi-modes slightly shifts when the value of $k_{y}$ varies so the driving frequencies in Fig. 11a do not exactly equal 5.52 for all 3 cases.) In contrast, a driving frequency close to this quasi-mode, for instance $\omega_{\mathrm{d}}=5.58$, results in a far 
Xi_y: $w=8.506$

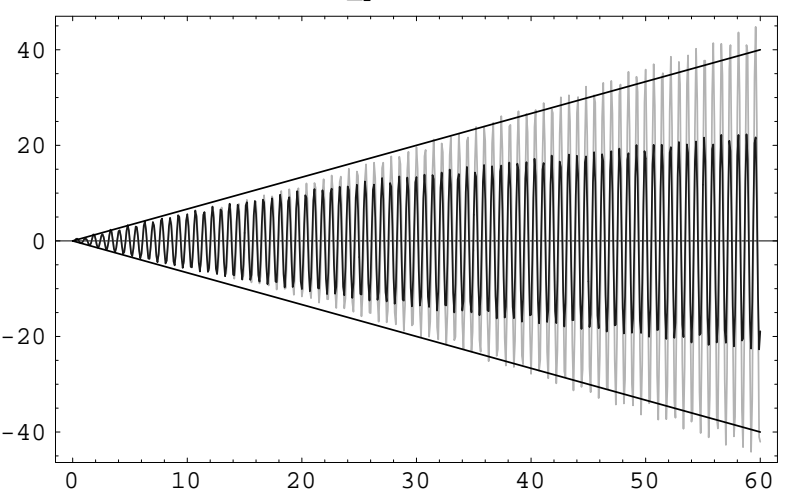

Xi_x: w=8.506

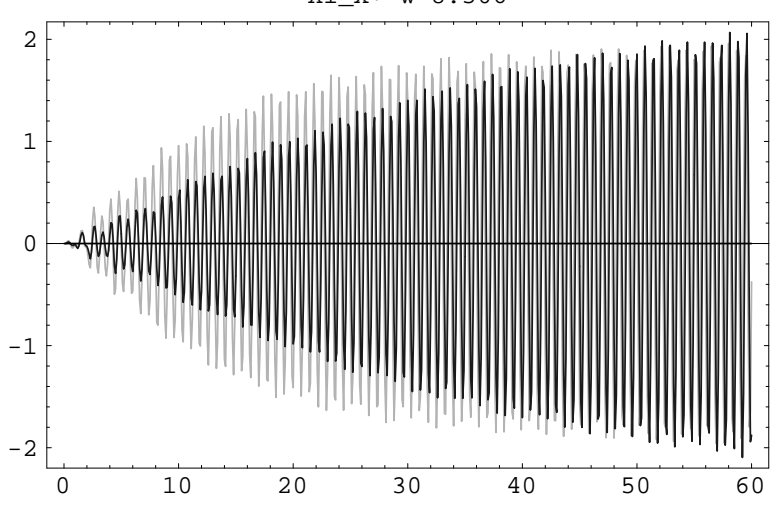

Fig. 10. Time evolution of the amplitude of $\xi_{y}$ and $\xi_{x}$ for $k_{y}=$ 0 (black), $k_{y}=1$ (dark-gray) and $k_{y}=2$ (light-gray) after driving with the quasi-mode frequency $\omega_{\mathrm{d}}=8.51$.

less efficient resonance growth as shown in Fig. 11b. The time at which saturation sets in strongly depends on the strength of the coupling and consequently on the value of $n$. Quasi-modes corresponding to higher values of $k_{z}$ (larger $n$ ) have a weaker coupling to Alfvén modes than $n=1$-modes. That is why the maximal efficiency in the resonance growth is attained much earlier after driving with the first harmonic $\omega \approx 5.52$ than with higher harmonics, like for example $\omega \approx 8.51$ (Fig. 10).

\section{Summary}

In the present investigation we focussed on the importance of coupled Alfvén-fast waves (quasi-modes) for wave dissipation in coronal loops driven by azimuthally polarized footpoint motions. As a first step to the investigation of a realistic random driver (see Paper II), we imposed monochromatic footpoint motions at the base of the loop model. First we study torsional Alfvén waves $\left(k_{y}=0\right)$ which do not couple to fast waves, so that the different magnetic surfaces live in perfect isolation and Alfvén waves can only be excited directly at the photospheric base. We showed that resonant absorption occurs at the magnetic surface where the Alfvén frequency matches the driving frequency; in the neighbouring surfaces of the resonance, secondary peaks are formed due to phase-mixing.
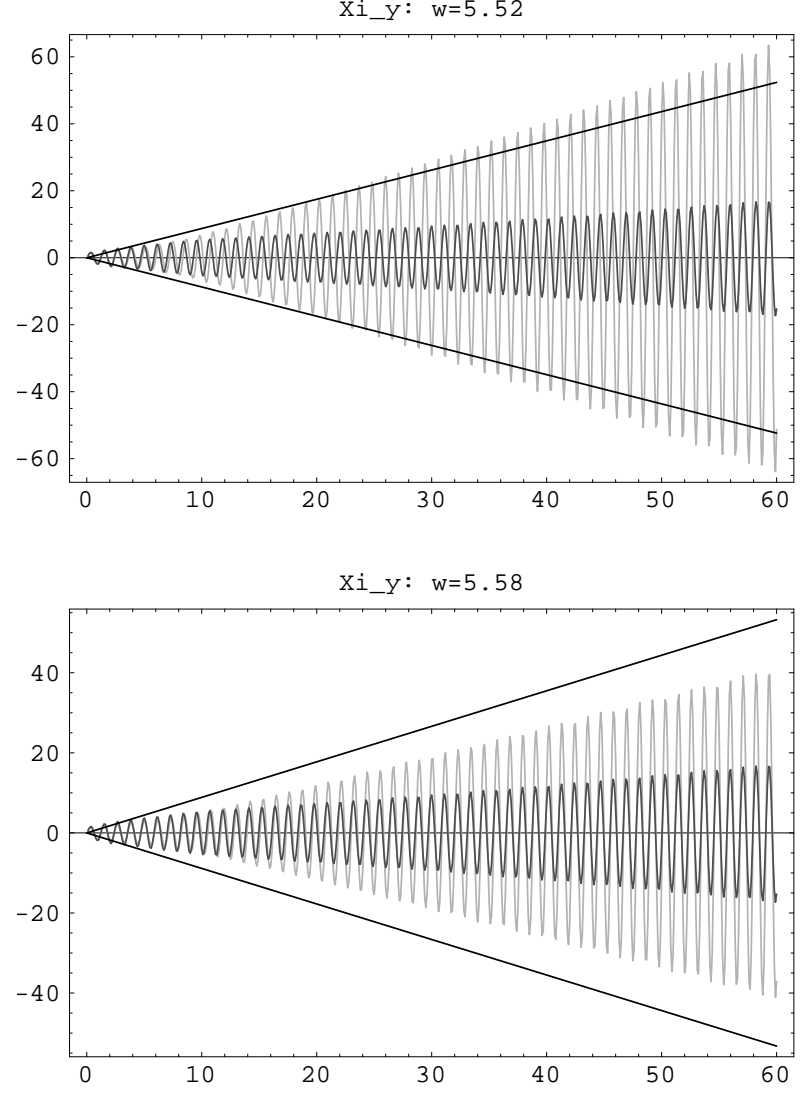

Fig. 11. Time evolution of the amplitude of $\xi_{y}$ for $k_{y}=0$ (black), $k_{y}=1$ (dark-gray) and $k_{y}=2$ (light-gray) with $\omega_{\mathrm{d}}=$ quasi-mode frequency (top) and $\omega_{\mathrm{d}}=5.58$ (bottom).

The amplitude of these peaks shows typical beats in time, as found analytically by e.g. Berghmans \& De Bruyne (1995). Since we took into account all possible values of $k_{z}$, cases were found where resonant peaks appeared at multiple locations, even though the loop was driven by periodic motions.

When coupling is taken into account, the behaviour of the MHD waves depends strongly on the driving frequency and the value of $k_{y}$. When $\omega_{\mathrm{d}}$ is close to a quasi-mode, the resonance built up at the location where $\omega_{\mathrm{d}}$ equals the local Alfvén frequency is much more pronounced than for a random value of $\omega_{\mathrm{d}}$ and the length scales of the resonance decrease at a higher rate.

On the other hand, coupling also has an important influence on the time evolution of the Alfvén resonance. For driving frequencies far from any quasi-mode, coupling to fast MHD waves has a negative effect on the growth of Alfvén mode energy. For quasi-mode frequencies however, standing global waves are excited and the resonance is amplified by the interplay between fast and Alfvén waves. For a strong coupling, the stationary state for $\xi_{x}$ sets in earlier and the resonance growth soon becomes more efficient than in the uncoupled case.

From the results collected in this paper, we can conclude that resonant absorption and phase-mixing processes indeed provide the small length scales required 
for wave dissipation in coronal loops driven by azimuthally polarized footpoint motions. The inherent coupling of Alfvén waves and fast magneto-acoustic waves for nonaxisymmetric motions $\left(k_{y} \neq 0\right)$ can both improve or undermine the process since it results in two channels for exciting resonant Alfvén waves. Depending on the driving frequency and the value of $k_{y}$, these directly and indirectly excited Alfvén waves either amplify or oppose each other. Quasi-modes play a key role in these processes since they act as energy carrier waves and provide the best conditions for excitation.

However, for monochromatic drivers the need for finetuning remains an obstacle and is far from realistic. In addition only a few resonant layers can be formed which can never result in a globally heated coronal loop. Therefore it is important to investigate how the physics is altered when a random driver is imposed at the footpoints. In Paper II the present investigation is extended by taking into account this more realistic way of exciting the MHD waves involved in the heating process. In addition, we explain how these results are related to recent observations.

\section{References}

Beliën, A. J. C., Martens, P. C. H., \& Keppens, R. 1999, ApJ, 526,478

Berghmans, D., \& De Bruyne, P. 1995, ApJ, 453, 495

Berghmans, D., De Bruyne, P., \& Goossens, M. 1996, ApJ, 472,398

Berghmans, D., \& Tirry, W. J. 1997, A\&A, 325, 318

Cally, P. 1991, J. Plasma Phys., 45, 453

De Groof, A., \& Goossens, M. 2000, A\&A, 356, 724

De Groof, A., \& Goossens, M. 2002, A\&A, 386, 691 (Paper II)

Falconer, D., Moore, R., Porter, J., Gary, G., \& Shimizu, T. 1997, ApJ, 482, 519
Gomez, D. O. 1990, Fund. Cosmic Phys., 14, 131

Goossens, M. 1991, in Advances in Solar System MHD, ed. E. R. Priest, \& A. W. Hood (Cambridge University Press), 137

Goossens, M., \& De Groof, A. 2001, Phys. Plasmas, 8, 2371

Goossens, M., Ruderman, M. S., \& Hollweg, J. V. 1995, Sol. Phys., 157, 75

Heyvaerts, J., \& Priest, E. R. 1983, A\&A, 117, 220

Ionson, J. A. 1978, ApJ, 226, 650

Kuperus, M., Ionson, J. A., \& Spicer, D. 1981, ARA\&A, 19, 7

Mann, I. R., Wright, A. N., \& Cally, P. S. 1995, J. Geophys. Res., 100, 19441

Ofman, L., \& Davila, J. M. 1995, J. Geophys. Res., 100, 23427

Ofman, L., \& Davila, J. M. 1996, ApJ, 456, L123

Ofman, L., Klimchuk, J. A., \& Davila, J. M. 1998, ApJ, 493, 474

Parker, E. 1972, ApJ, 174, 499

Poedts, S., Beliën, A. J. C., \& Goedbloed, J. P. 1994, Sol. Phys., 151, 271

Poedts, S., \& Boynton, G. C. 1996, A\&A, 306, 610

Poedts, S., Goossens, M., \& Kerner, W. 1989, Sol. Phys., 123, 83

Poedts, S., \& Kerner, W. 1992, J. Plasma Phys., 47, 139

Roussev, I., Galsgaard, K., Erdélyi, R., \& Doyle, J. G. 2001, A\&A, 370, 298

Ruderman, M. S., Berghmans, D., Goossens, M., \& Poedts, S. 1997, A\&A, 320, 305

Steinolfson, R. S., \& Davila, J. M. 1993, ApJ, 415, 354

Tirry, W. J., \& Berghmans, D. 1997, A\&A, 325, 329

Tirry, W. J., Berghmans, D., \& Goossens, M. 1997, A\&A, 322, 329

Tirry, W. J., \& Goossens, M. 1996, ApJ, 471, 501

Tsuneta, S. 1996, in NATO ASI Ser. 418, Solar and Astrophysical Magnetohydrodynamic Flows, Fodele, Crete, June 11-22, 1995, ed. K. Tsinganos, 23

Van Ballegooijen, A. A. 1985, ApJ, 298, 421

Wright, A. N., \& Rickard, G. J. 1995, ApJ, 444, 458 